\title{
Far-Field High-Energy Diffraction Microscopy: A Non-Destructive Tool for Characterizing the Microstructure and Micromechanical State of Polycrystalline Materials
}

\author{
Jun-Sang Park, ${ }^{1 *}$ Xuan Zhang, ${ }^{2}$ Peter Kenesei, ${ }^{1}$ Su Leen Wong, ${ }^{3}$ Meimei Li, ${ }^{2}$ \\ and Jonathan Almer ${ }^{1}$ \\ ${ }^{1}$ X-ray Science Division, Argonne National Laboratory, 9700 S Cass Avenue, Lemont IL 60439 \\ ${ }^{2}$ Nuclear Engineering Division, Argonne National Laboratory, 9700 S. Cass Ave., Lemont IL 60439 \\ ${ }^{3}$ Max-Planck-Institut fur Eisenforschung GmbH, Max-Planck-Straße 1, 40237, Düsseldorf, Nordrhein-Westfalen, Germany \\ *parkjs@aps.anl.gov
}

\begin{abstract}
A suite of non-destructive, three-dimensional (3D) microscopy techniques using high-energy synchrotron X-rays has been developed over the past decade. These have been used to characterize microstructures and micromechanical states of various polycrystalline materials. Several sample environments compatible with these 3D microscopy techniques have come on-line to enable in situ measurements. This article describes the far-field high-energy diffraction microscopy (FF-HEDM) technique implemented at the 1-ID beamline of the Advanced Photon Source. Examples presented illustrate how FF-HEDM can be used to deepen our understanding of structureproperty-processing relationships in polycrystalline materials.
\end{abstract}

\section{Introduction}

The ability to non-destructively map three-dimensional (3D) microstructures and their evolution following external stimuli such as load or heat is of great interest to the materials science and engineering community. As described in a recent review article [1], microscopy techniques employing visible light or electron beams have been used extensively to characterize material systems so that their properties can be predicted from their microstructures. With these approaches, a polycrystalline sample is typically mapped in $2 \mathrm{D}$, and a series of $2 \mathrm{D}$ maps are combined to create a $3 \mathrm{D}$ view of the microstructure. Taking advantage of various contrast mechanisms, tomography techniques have also been used to map microstructures in 3D [2]. While tomography techniques are non-destructive and provide views of the complex internal structures of materials, these methods are typically insensitive to crystallographic orientations in the grain structure that is often related to material properties [3].
In the past decade, several non-destructive 3D microscopy techniques using high-energy synchrotron X-rays have come on-line [4-8]. These techniques are capable of characterizing the microstructure of a relatively large polycrystalline aggregate at the grain length scale with sensitivity to crystallographic orientation. Combined with suitable experimental apparatus, these techniques can be performed in situ; thus, the evolution of a polycrystalline aggregate microstructure can be tracked while external stimuli such as load, electric current, or heat are applied [9-12]. This experimental capability opens new opportunities for scientists and engineers to deconvolve and understand the complex relationships between processing history, grain structure, and component performance. Experimental data obtained from these techniques can be used to validate and calibrate physically based material models crucial to efforts such as Integrated Computational Materials Engineering (ICME) and the Materials Genome Initiative (MGI).

In this article we describe the far-field high-energy diffraction microscopy (FF-HEDM) setup at the 1-ID beamline of the Advanced Photon Source (APS) at Argonne National Laboratory. Examples are presented of how FF-HEDM data can be used to validate physically based models and to understand the intricate relationship between structure, processing, and properties.

\section{Far-Field High-Energy Diffraction Microscopy at the APS}

High-energy synchrotron radiation. X-rays from synchrotrons with energies greater than $50 \mathrm{keV}$ have a unique set of characteristics that make them an ideal probe for characterizing

Table 1: HEDM techniques available at the APS 1-ID beamline [19].

\begin{tabular}{|l|l|l|}
\hline Experimental Technique & Spatial Resolution & Remarks \\
\hline $\begin{array}{l}\text { Near-field } \\
\text { high-energy diffraction } \\
\text { microscopy } \\
\text { (NF-HEDM) }\end{array}$ & $\begin{array}{l}\sim 1 \mu \mathrm{m} \text { spatial resolution; } \\
0.1^{\circ}-0.01^{\circ} \text { angular } \\
\text { resolution }\end{array}$ & $\begin{array}{l}\text { Area detector }(1.5 \mu \mathrm{m} \text { square pixels covering } \sim 3.1 \mathrm{~mm} \times \sim 3.1 \mathrm{~mm} \\
\text { area) placed } \sim 10 \mathrm{~mm} \text { away from the sample. Analogous to } \\
\text { non-destructive 3D electron backscatter diffraction view of a } \\
\text { polycrystalline aggregate using a line focused beam [13-14]. } \\
\text { Does not provide strain information. }\end{array}$ \\
\hline $\begin{array}{l}\text { Far-field } \\
\text { high-energy diffraction } \\
\text { microscopy } \\
\text { (FF-HEDM) }\end{array}$ & $\begin{array}{l}\sim 10 \mu \mathrm{m} \text { spatial resolution; } \\
0.1^{\circ}-0.01^{\circ} \text { crystallographic } \\
\text { orientation resolution; } 10^{-4} \\
\text { strain resolution }\end{array}$ & $\begin{array}{l}\text { Area detector }(200 \mu \mathrm{m} \text { square pixels covering } \sim 410 \mathrm{~mm} \times \sim 410 \mathrm{~mm} \\
\text { area) placed } \sim 1 \mathrm{~m} \text { away from the sample. Non-destructive 3D map } \\
\text { of the centers of masses, crystallographic orientations, radii, and } \\
\text { elastic strain tensors of the grains in a polycrystalline aggregate [8]. }\end{array}$ \\
\hline $\begin{array}{l}\text { Very-far-field high-energy } \\
\text { diffraction microscopy } \\
\text { (VFF-HEDM) }\end{array}$ & $\sim 0.01^{\circ}$ angular resolution & $\begin{array}{l}\text { Area detector }(\sim 60 \mu \mathrm{m} \text { pixels covering } \sim 50 \mathrm{~mm} \times \sim 30 \mathrm{~mm} \text { area) } \\
\text { placed } \sim 5 \mathrm{~m} \text { away from the sample. Non-destructive reciprocal space } \\
\text { mapping of individual grains in a polycrystalline aggregate [18]. }\end{array}$ \\
\hline
\end{tabular}


(a)

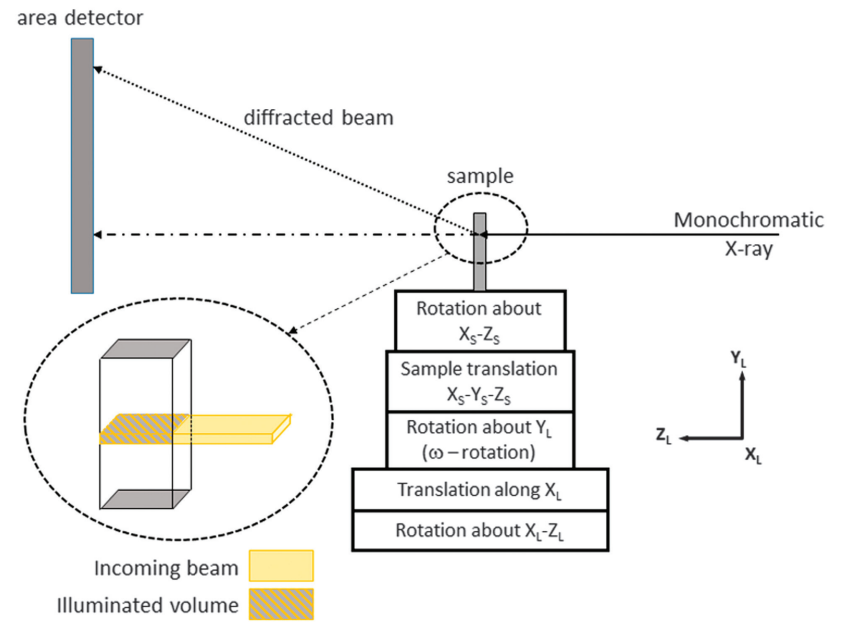

(b)

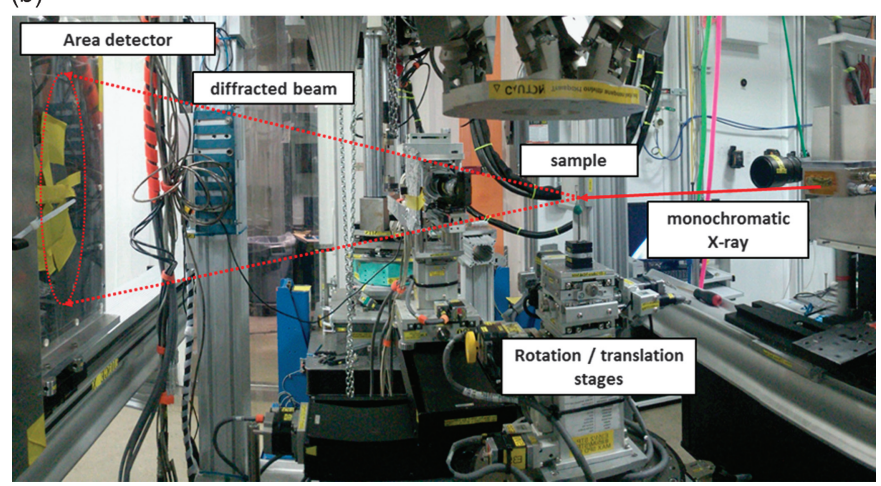

Figure 1: Illustrations of the FF-HEDM setup at the APS 1-ID beamline. The subscript $S$ refers to the translations and rotations in the sample coordinate system. The subscript $L$ refers to the translations and rotations in the laboratory coordinate system. (b) Panoramic view of the FF-HEDM setup at the APS 1-ID-E beamline.

polycrystalline materials. Penetration depth through materials can be on the order of millimeters for most engineering alloys and materials, which is orders of magnitude larger than most laboratory $\mathrm{X}$-ray sources. Small diffraction angles combined with the use of area detectors, strategically placed at various distances downstream from the sample (transmission geometry), allow us to characterize features in a material at various length scales from nanometers (using small- or wide-angle X-ray scattering techniques) to $\mu \mathrm{m}$ (using HEDM). Furthermore, when combined with high-energy $\mathrm{X}$-ray tomography, a wide range of features at various length scales in a material can be characterized. With recent advances in focusing optics suitable for high-energy X-rays, $\sim 1-10 \mu \mathrm{m}$ spot size is routinely achievable at a wide range of energies [13-14], and sub- $\mu \mathrm{m}$ spot size also has been achieved [15]. The spot size is anticipated to improve significantly as emittance of the electron beam in the storage ring is reduced with the introduction of fourth-generation synchrotron sources. [16]. Finally, vacuum environments, used to minimize air scattering of electron beams and necessary for experiments with low-energy X-rays, are not necessary. This means that a variety of in situ or in operando environments [9-12,17] can be developed with relative ease.

Experimental setup. Our setup uses high-energy X-rays in transmission geometry to interrogate a polycrystalline aggregate of material. The sample is rotated about an axis with respect (a)
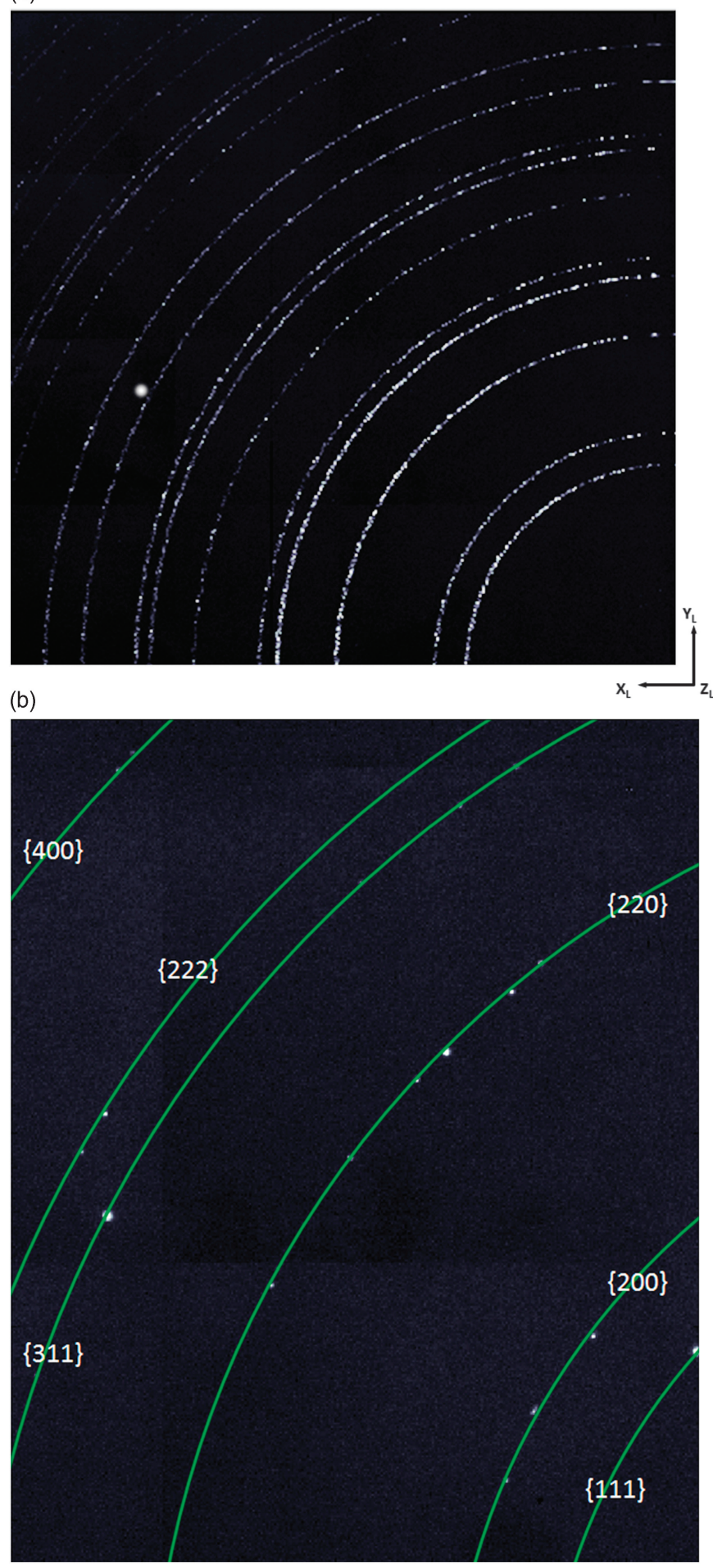

Figure 2: Example of a FF-HEDM diffraction pattern taken at a wavelength of $0.017286 \mathrm{~nm}(71.671 \mathrm{keV})$ using a well-annealed steel sample with face-centeredcubic (fcc) crystal symmetry. The sample-to-detector distance was $755 \mathrm{~mm}$. (a) Example of summed area detector diffraction pattern images taken between $-180^{\circ}$ and $-120^{\circ}$ at $\omega=0.25^{\circ}$ intervals for one illuminated volume. The figure only shows a quadrant. Each ring on the image corresponds to a diffracting family of crystallographic planes. Information within individual frames is typically very sparse. (b) Magnified view of a typical frame of an FF-HEDM image set. Green curves illustrate the theoretical location of the Debye-Scherrer rings. Strains in the crystal grains caused the diffraction spots to deviate from the theoretical Debye-Scherrer ring position. The integrated intensity of a diffraction spot is related to the volume of the diffracting grain. 


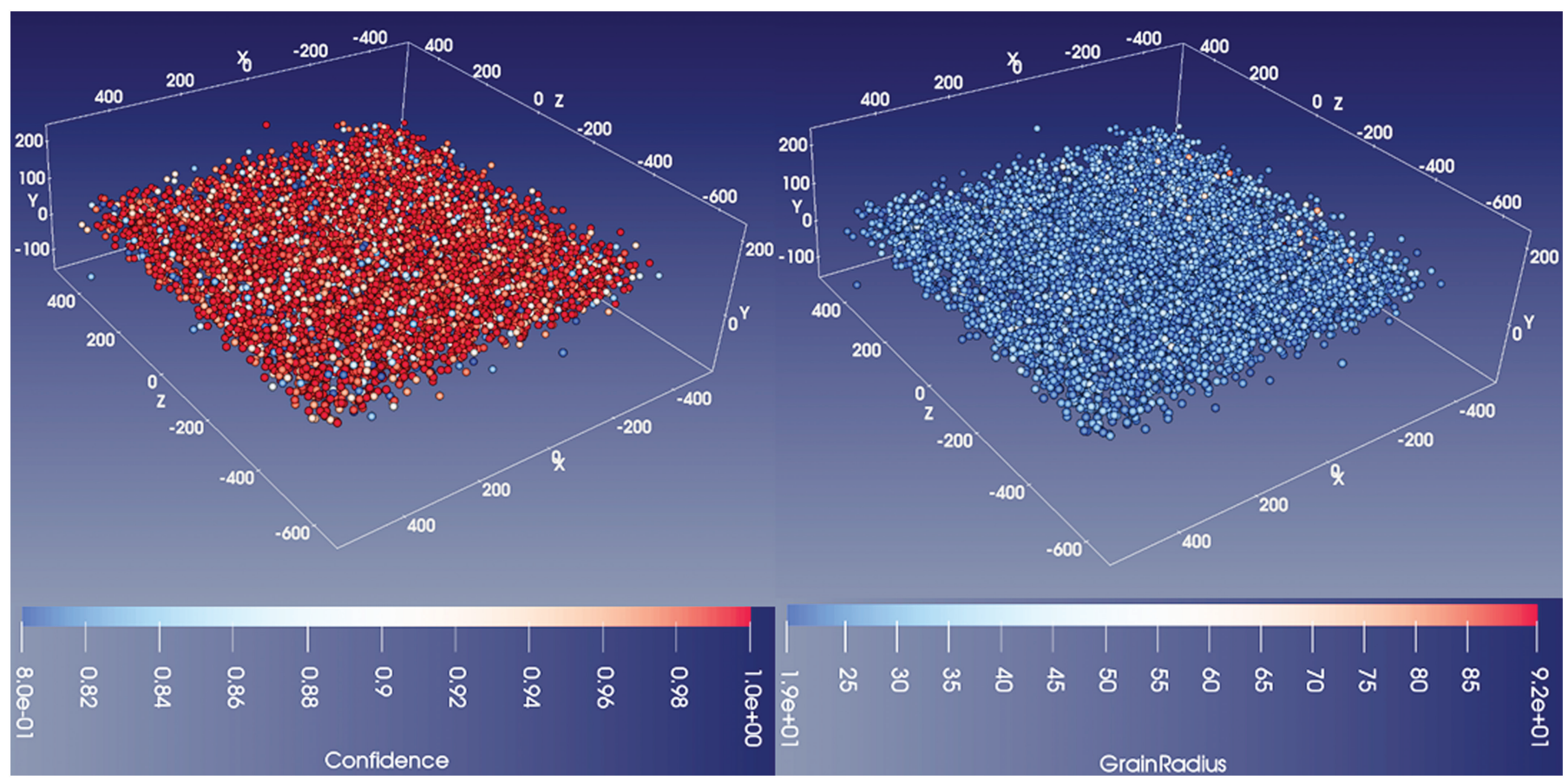

Figure 3: An example of grain map obtained by FF-HEDM from a stainless steel sample. The sample was $1 \mathrm{~mm} \times 1 \mathrm{~mm}$ in cross section. The wavelength of the $\mathrm{X}$-rays was $0.017286 \mathrm{~nm}(71.671 \mathrm{keV})$. The beam size was $2 \mathrm{~mm} \times 0.1 \mathrm{~mm}$ (along $X_{L}$ and $Y_{L}$ respectively). The diffraction data were analyzed by MIDAS. The location of each sphere corresponds to the center of mass location of the found grains. Axes are in $\mu \mathrm{m}$. Associated with each grain are its crystallographic orientation and lattice strain tensor. (Left) Color shows completeness/confidence. (Right) Color shows grain radius $(\mu \mathrm{m})$.

(a)

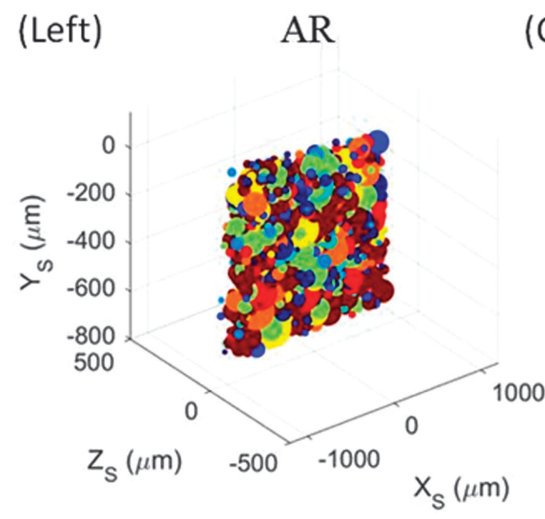

(b)

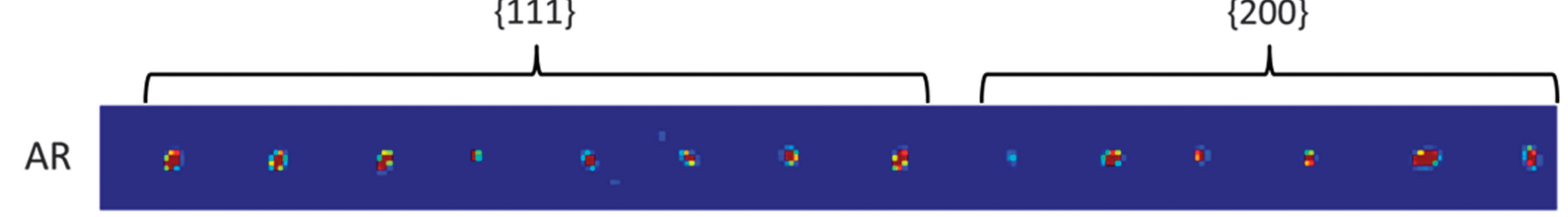

irr

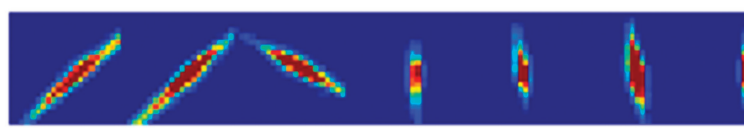

irrtann

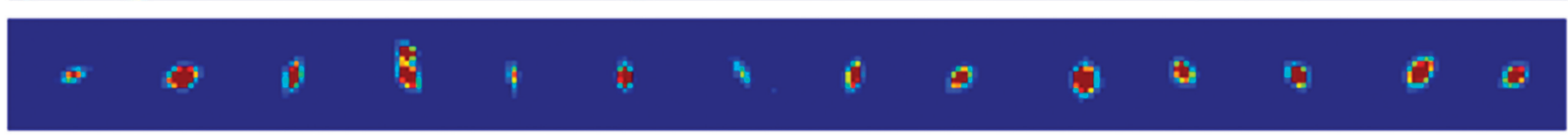

Figure 4: Comparison of the FF-HEDM results for the as-received (AR), irradiated (irr), and irradiated and annealed (irr + ann) samples. (a) Center of mass maps for the AR sample (left), the neutron-irr sample (center), and the irr + ann sample (right). Figures replicated from [19]. (b) Diffraction spots from single grains in the three samples. 
(a)

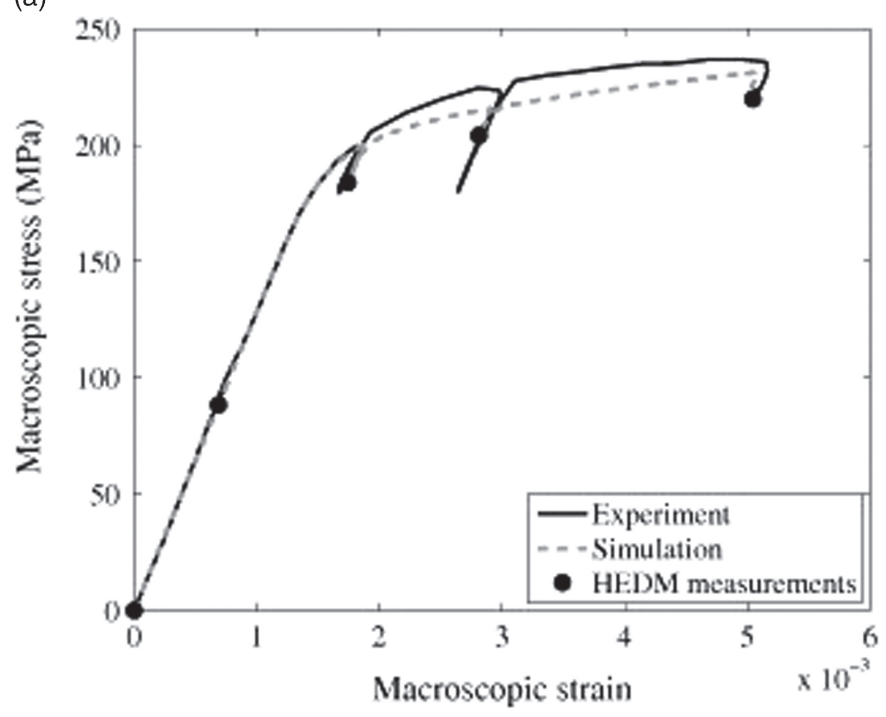

(b)

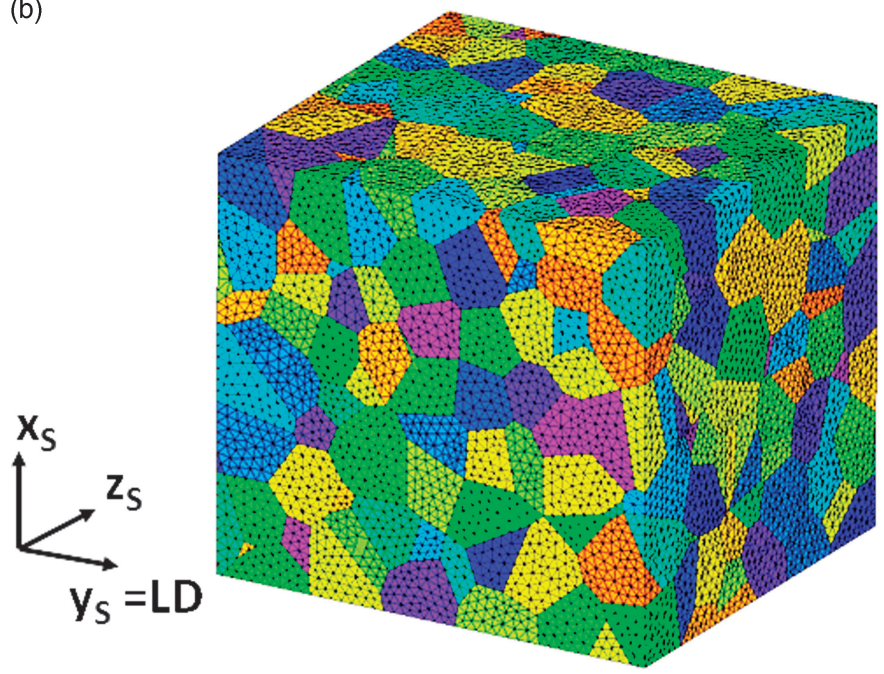

Figure 5: (a) Macroscopic stress-strain curve of the copper alloy investigated in [23]. (b) View of the virtual polycrystal created using the orientations of the constituent grains obtained from FF-HEDM measurements. Colors denote grains of different orientations. Figures replicated from [23] with permission of Cambridge University Press.

to the incoming X-rays. When a particular crystallographic plane satisfies the Bragg diffraction condition, the diffracted X-ray is recorded on an area detector. Currently, three flavors of high-energy diffraction microscopy techniques exist. Table 1 summarizes the HEDM techniques available to the general users at the APS 1-ID-E beamline. Similar capabilities also exist at the other synchrotron facilities around the world, with various spatial and angular resolution capabilities. As indicated in Table 1, the FF-HEDM technique is insensitive to the shape of the grain illuminated by $\mathrm{X}$-rays. It is only sensitive to the crystallographic orientation and spatial location of the grain. Additionally, it is sensitive to the elastic strain of the grain. The ability to measure elastic strain, albeit grain averaged, is useful by itself and when combined with other HEDM techniques and tomography techniques. Our setup at the APS is capable of conducting in situ multimodal experiments to obtain information about the evolution of the microstructure and micromechanical state of polycrystals.
Figure 1a shows a schematic of the key components for the FF-HEDM technique, and Figure $1 \mathrm{~b}$ shows a panoramic view of the ex situ setup in the 1-ID-E hutch of the APS. High-energy monochromatic X-rays illuminate a volume of polycrystalline sample, which is placed on top of rotation and translation stages. The width of the beam is typically larger than the maximum width of the sample, while the height of the beam is adjusted to be as large as possible without allowing diffraction spots from the constituent grains to overlap. The beam size is defined either by slits or refraction-based high-energy $\mathrm{X}$-ray focusing optics that allow a $1 \mu \mathrm{m}$ beam size in the $\mathrm{Y}_{\mathrm{L}}$ direction [13-14]. For most samples, a double-Laue monochromator is used (energy range: $40-130 \mathrm{keV}$; energy bandwidth $(d E / E) \approx 10^{-3}$ ), but for samples requiring increased reciprocal-space resolution (for example, after large plastic deformation where the diffraction spots are smeared significantly or substructures have formed in the grains), a secondary high-resolution monochromator can be used $\left(d E / E \approx 10^{-4}\right)$ [20-21]. Rotation is performed about the $Y_{L}$ axis $(\omega$ rotation). A set of translation and rotation stages are used to align the $\omega$ rotation axis to be perpendicular to the incident $\mathrm{X}$-ray beam propagating on the $\mathrm{X}_{\mathrm{L}}-\mathrm{Z}_{\mathrm{L}}$ plane. Often, the sample has its own natural coordinate system (for example, a sample extracted from a rolled plate), and rotation and translation stages are used to align the sample with respect to the beam. Both ex situ and in situ experiments are possible; for the latter, thermo-mechanical loading may be used to follow the evolution of microstructure and micromechanical state on a grain-by-grain basis.

Diffraction patterns and analysis. Diffraction patterns are typically taken at $0.25^{\circ}$ intervals as the sample is rotated between $-180^{\circ}$ and $+180^{\circ}$ in $\omega$. When a crystallographic plane from a constituent grain in the illuminated volume satisfies the diffraction condition, the corresponding diffraction spot is recorded on the area detector. A GE Revolution 41RT detector [22], with $409.6 \mathrm{~mm} \times 409.6 \mathrm{~mm}$ active area and $0.2 \mathrm{~mm} \times 0.2 \mathrm{~mm}$ pixel pitch, is typically used for the measurements, with the detector placed $\sim 1 \mathrm{~m}$ downstream of the sample. In some cases, four detectors are arranged in a diamond pattern for enhanced resolution and coverage [23]. At these detector distances, information related to the detailed shapes of the diffracting grains is lost, but their centers of mass (COM), crystallographic orientations, and lattice strain tensors can be obtained. Figure 2a shows an example of an FF-HEDM diffraction pattern, where distinct diffraction spots can be seen.

Diffraction patterns like the one shown in Figure $2 \mathrm{~b}$ are used to determine the COM, orientation, and lattice strain of the constituent grains through a processing called indexing. The indexing methods are described extensively in various references [4, 24-25]. In general, the following steps are taken and iterated: (1) Identify and isolate all the diffraction spots from the raw diffraction pattern. Each spot can be parameterized by $\mathrm{X}_{\mathrm{L}}, \mathrm{Y}_{\mathrm{L}}$, and $\omega$. (2) Search in orientation space for crystallographic orientations that yield the observed diffraction spots. (3) For a particular crystallographic orientation found in the previous step and associated spots, search in physical space $\left(\mathrm{X}_{S^{-}} \mathrm{Y}_{\mathrm{S}}-\mathrm{Z}_{\mathrm{S}}\right)$ and strain space to obtain the $\mathrm{COM}$ and strain tensor associated with the orientation.

There are several analysis tools available to the general public [24-26]. The APS staff, in collaboration with the 

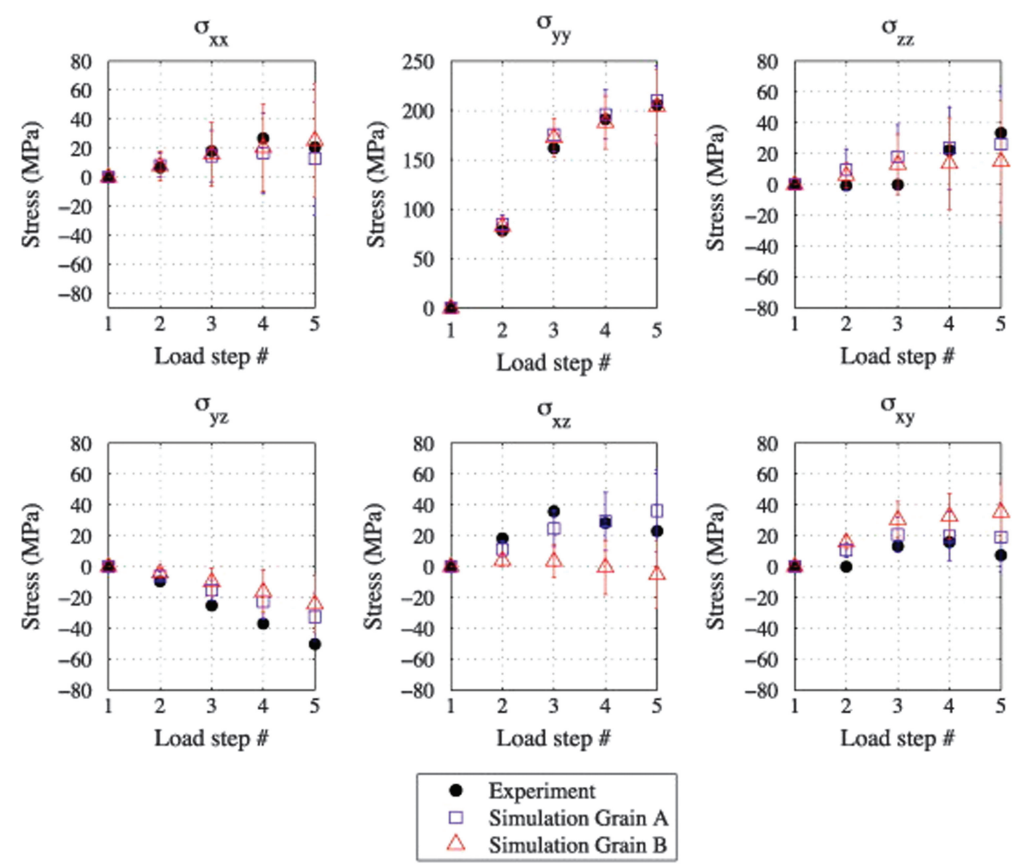

Figure 6: Stress components of a grain determined from FF-HEDM and simulations. The error bars on the experimental stresses are deduced from experimental strain uncertainty. The error bar on the simulation stresses were obtained by calculating the standard deviation of the stress for all elements that belong to the grain in the virtual polycrystal. Figure replicated from [23] with permission of Cambridge University Press.

user community, have recently developed a package titled Microstructural Imaging using Diffraction Analysis Software (MIDAS) that takes advantage of high-performance computing [26-27] so that experimenters can quickly map 3D volumes, sometimes rapidly enough to steer in situ experiments (for example, to optimize the amount of thermo-mechanical load to apply prior to or just after crack initiation). Figure 3 shows some examples of microstructure information obtained from analyzing the raw FF-HEDM data. In this figure, the grain COMs are illustrated by the location of the spheres in space. The colors of the spheres denote information related to the grains.

Experimental uncertainty. In a typical configuration, an illuminated volume that consists of approximately 1,000 grains with a uniform size distribution, uniform crystallographic texture, and moderate levels of plastic deformation can be reliably characterized with relative ease. As the data analysis procedure involves identifying and isolating the diffraction spots, a larger number of grains in the aggregate or strong crystallographic texture increase the probability of spot overlap. The dynamic range of the area detector limits the grain size distribution since the integrated intensity of a diffraction spot from a grain is proportional its volume. High levels of deformation typically generate substructures and mosaicity, which cause the diffraction spots to smear, and smeared diffraction spots are more difficult to parameterize with XL, YL, and $\omega$.

In our typical setup, the spatial and orientation uncertainties are on the order of $10 \mu \mathrm{m}$ and $0.1^{\circ}$, respectively. The strain uncertainty is on the order of 0.0001 . The performance of the method is highly dependent on setup and the state of the sample, and these values are provided only as guidance. If the state of the illuminated volume deviates from ideal conditions, these uncertainties will suffer and the analysis can become more challenging.

\section{Application Examples of FF-HEDM Data}

Characterization of neutron-irradiated steel. While

the effects of neutron irradiation on structural steels used in nuclear energy applications have been studied extensively using conventional microscopy techniques, grain-level investigations within bulk samples have been, until recently, impossible because of the difficulties in handling activated materials. Zhang et al. [28] investigated the effect of neutron irradiation on the microstructure of a high-temperature ultrafine-precipitated-strengthened (HT-UPS) austenitic stainless steel ex situ.

This HT-UPS austenitic stainless steel is a candidate for structural and cladding applications in the next-generation nuclear reactors because of its improved creep resistance and potentially better radiation resistance over the traditional Type 316 stainless steels [29-31]. In the work by Zhang et al. [28], effects of neutron irradiation and post-irradiation annealing on grain-scale microstructure of a solution annealed HT-UPS steel were investigated using FF-HEDM technique at the APS 1-ID-E beamline. Three disk-shaped samples were investigated: unirradiated as-received (AR) steel, neutron irradiated to 3 displacements per atom (dpa) at $500^{\circ} \mathrm{C}$ (IRR), and the same irradiated condition but subsequently annealed at $600^{\circ} \mathrm{C}$ for 1 hour (IRR+ANN). The FF-HEDM experiments were conducted using $\mathrm{X}$-ray energy of $65.35 \mathrm{keV}$. The beam size was $2 \mathrm{~mm}\left(\right.$ along $\mathrm{X}_{\mathrm{L}}$ ) $\times 0.2 \mathrm{~mm}$ (along $\mathrm{Y}_{\mathrm{L}}$ ) to fully illuminate the sample cross section. A set of FF-HEDM diffraction patterns were acquired in sweeping mode with $0.5^{\circ}$ increments in $\omega$ over the range of $-180^{\circ}$ to $+180^{\circ}$. After acquiring a set of diffraction patterns for a layer illuminated by the beam, the sample was translated along $\mathrm{Y}_{\mathrm{S}}$ by $0.2 \mathrm{~mm}$ to have another layer measured. For each sample, four layers were measured in total, yielding a total interrogated volume of approximately $0.3 \mathrm{~mm}^{3}$. The total interrogated volume contained several hundreds of grains suitable for statistical analysis. Analysis of the FF-HEDM data was carried out using MIDAS software and diffraction spots from the $\{111\}$ and $\{200\}$ lattice planes.

Figure 4a shows the FF-HEDM maps of the three samples. Each grain is represented by a sphere. The size of a sphere is proportional to the volume of the corresponding grain. Locations of the spheres correspond to the COMs of the grains in the respective samples. The color of the sphere corresponds to the completeness of the grain. Completeness is defined as the ratio of the number of measured diffraction spots during an FF-HEDM experiment and the number of expected diffraction spots for a particular grain. Completeness of 1.0 indicates that all the diffraction spots are correctly found in the measured data. This metric is used as a measure of how confident we are with the identified grain [24]. In total, 943 grains were found for the AR sample with an average grain radius of $44 \mu \mathrm{m}$ and an average completeness of 0.90 , contributing to over $90 \%$ of the total volume. In contrast, 843 grains were detected in the IRR sample with an average radius of $41 \mu \mathrm{m}$ and an average completeness of 0.84 , making up to only $75 \%$ of the total volume. This comparison indicates that while the neutron irradiation does not change the grain size significantly, it introduces damage to the material as indicated by the decrease in both 


\section{We're Really BIG on TINY}

We make Tiny Holes in molybdenum, platinum and other materials.

Diameters from 10um all the way down to $100 \mathrm{~nm}$.

We make Tiny Tools in diamond, glass and other materials, all supporting linear dimensions less than 10 um for imaging, metrologic probes, nanomechanical testing and other applications. Fully turned geometries of submicron radii are feasible.

We make Tiny Marks as micrographic inscriptions in a great variety of materials for branding security, tracking, optoelectronic testing and other purposes.

We certainly can't predict what you might want these tiny items for, but with over twenty years of experience making them, we invite you to get in touch with us. We may be able to put a demonstration piece or prototype into your hands within a matter of just weeks.

We're really big on tiny things.

Norsam Technologies, Inc. | www.norsam.com | Phone 505.984. 1133
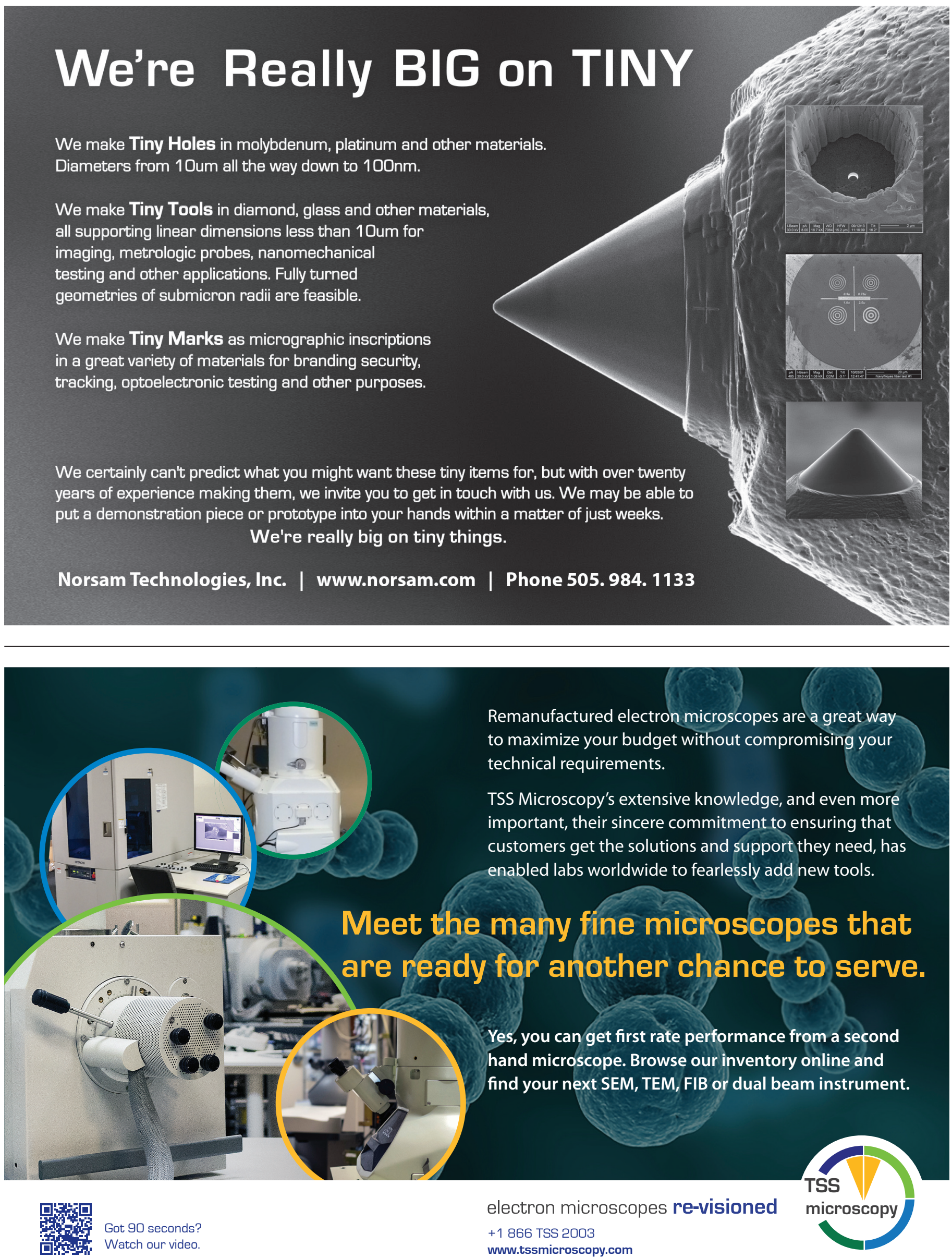


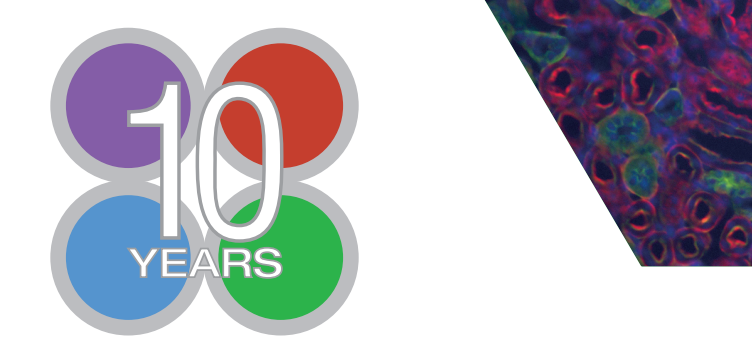

lumencor

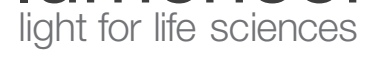

\section{IDEAL}

ILLUMINATION PLATFORM

for OEM Bioanalytical Instruments

\section{PARTNER WITH LUMENCOR FOR HIGH PERFORMANCE LIGHTING...}

TAILORED TO YOUR EQUIPMENT NEEDS.

Would your scanner or imaging equipment benefit from more optical power, faster switching speeds, greater stability and better reproducibility? Engage Lumencor for a customized light engine, tailored to meet the spectral, spatial and temporal needs of your imaging and/or bioanalysis instrument. Lumencor's lighting designs can be readily implemented for fluorescence and transmittance, when fast prototyping is needed to reduce time to market. Our state-of-the-art facilities support volume manufacturing.
Leverage our expertise and experience for your next new product or to develop instrument upgrades:

- Engineering and Design

- Optics, Mechanics, Electronics, Fluidics and Software Capabilities

- Prototyping

- Manufacturing

- Test and Measurement

- Certification and Documentation

Our team supports early stage development through small or large scale production. Discover our capabilities for design innovation from early concept to final deliverables. 
completeness and total volume recovered. Post-irradiation annealing removes much of the irradiation-induced damage. This is indicated by an average completeness of 0.89 and a total reconstructed volume of over $90 \%$ in the IRR + ANN sample, values that are close to those measured in the AR sample. The average grain size of the IRR + ANN sample is $40 \mu \mathrm{m}$, similar to those measured in the other two samples.

Individual grains in an aggregate can be examined in more detail and grains in various material states can be compared. Figure $4 \mathrm{~b}$ shows a series of diffraction spots from an interior grain in each sample. Their radii were all approximately $30 \mu \mathrm{m}$, and they all had completeness of 1.0. Compared to the grain from the AR sample, the grain from the IRR sample shows significantly broadened diffraction spots in both the radial direction and the azimuthal direction, indicating that irradiation introduces various types of damage. Furthermore, this indicates that neutron irradiation introduces damage rather uniformly in the material as opposed to the selective smearing seen in crystals that have undergone deformation [32]. The spots sharpen after annealing, providing solid evidence that post-irradiation annealing recovers the crystal structure of the grains.

Validation of crystal plasticity models. Decreasing the need for extensive physical experiments to test and certify new materials is a major goal in materials design and engineering (for example, the aforementioned ICME and MGI efforts). One key component in these efforts is being able to validate computer models with experimental data obtained at appropriate length scales. An example of how FF-HEDM can aid this effort is provided by Wong et al. [23] who combined FF-HEDM with in situ thermomechanical loading to critically test a crystal plasticity model implemented using a finite element formulation. In this work, a uniaxial tension sample made from a high-strength copper alloy $(\mathrm{Cu}-\mathrm{Cr}-\mathrm{Zn})$ with a $2 \mathrm{~mm} \times$ $2 \mathrm{~mm}$ cross section was subjected to monotonic uniaxial tension. The material was composed of equiaxed grains approximately $150 \mu \mathrm{m}$ in diameter. At pre-defined macroscopic loads along the uniaxial stress-strain curve, the loading was paused, and FF-HEDM measurements were performed to track the evolution of orientation and stress on a grainby-grain basis. Figure 5a shows the Cambridge University Press. macroscopic stress strain curve and locations where FF-HEDM measurements were conducted. The FF-HEDM experiments were conducted at $80.73 \mathrm{keV}$ with a $2 \mathrm{~mm} \times 0.1 \mathrm{~mm}$ beam to illuminate a volume of material in the gauge section. Then the sample was translated along $Y_{L}$ to increase the volume of material interrogated. From these FF-HEDM experiments, the crystallographic orientations and the lattice strain tensors of the constituent crystals were obtained. Using these crystallographic orientations, a virtual polycrystal was constructed as shown in Figure 5b. The virtual polycrystal was used to test the crystal plasticity model. Modelling details are presented in [33].

The validation of the model is shown in Figure 6, which shows the stress and its evolution for a grain determined from FF-HEDM experiments. It also shows the stress and its

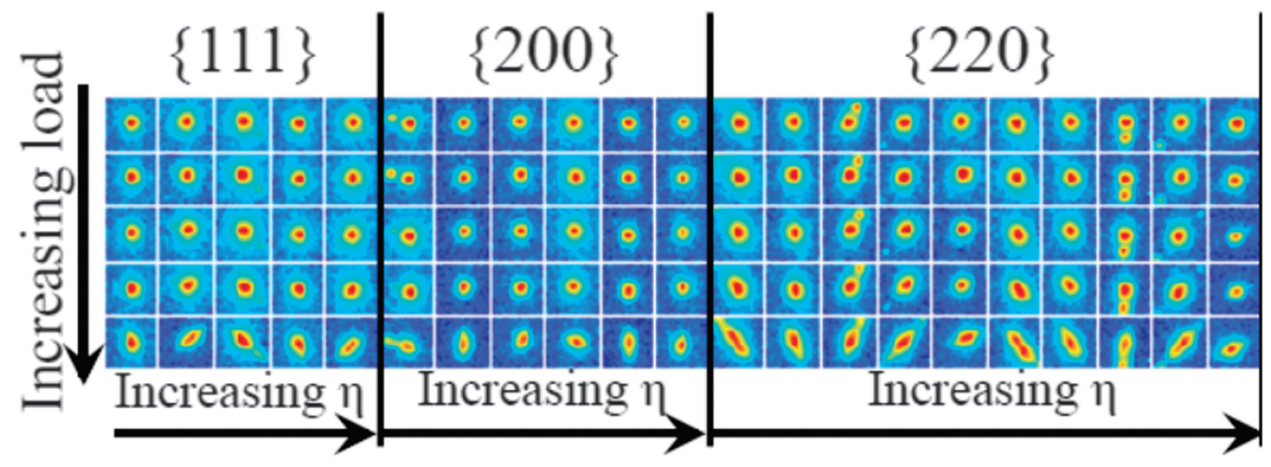

(a) Experimentally measured diffraction spots

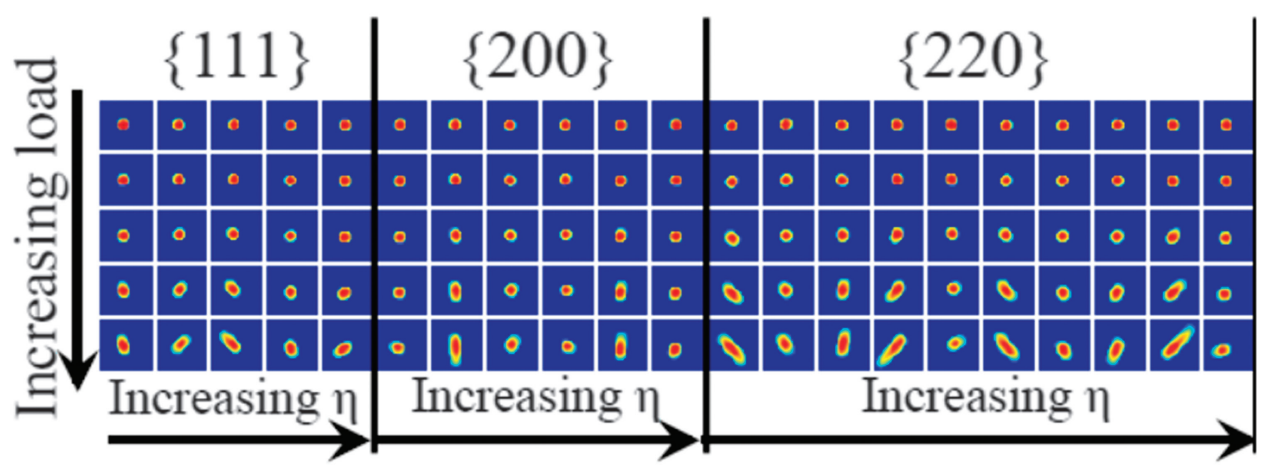

(b) Simulated diffraction spots from Grain A

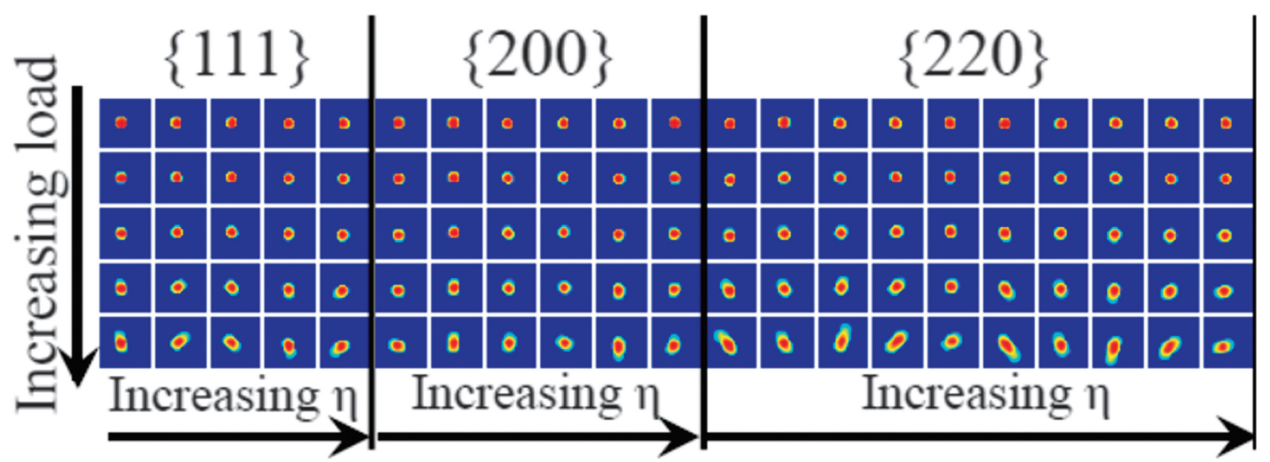

(c) Simulated diffraction spots from Grain B

Figure 7: Comparison between (a) the diffraction spots measured using FF-HEDM and corresponding synthetic diffraction spots from finite element simulation for (b) grain A and (c) grain B in the polcrystalline aggregate. Here, $\eta$ is the azimuthal angle where the spot is recorded on the detector. Figure replicated from [23] with permission of 
evolution for two grains located in the virtual sample with identical crystallographic orientation as the grain determined from FF-HEDM experiments. Two virtual grains were used to examine the effect of the neighborhood on the crystal stresses. The experimental stresses were computed from the measured lattice strain tensor and anisotropic Hooke's law, and these were compared to the simulation stresses. Figure 6 shows that the grain is in a multi-axial stress state even though the applied load on the polycrystalline aggregate was uniaxial. Furthermore, the two virtual grains show significant differences (xz component of stress for example) indicating that the neighborhood plays a significant role in determining the stress state of a crystal in an aggregate.

Figure 7 shows the evolution of diffraction spots associated with the grains shown in Figure 6. Similar to the diffraction spots shown in Figure 4b, the experimental diffraction spots for the copper grain also show significant changes. However, unlike the diffraction spots shown in Figure 4b, some diffraction spots smear significantly with applied load, while others do not. This indicates that smearing from deformation is perhaps more dependent on the crystallographic orientation of the crystal, whereas neutron irradiation and its damage may be less sensitive to the crystallographic orientation. Simulation results also show similar trends to those seen in the experimentally measured diffraction spots with subtle differences indicating the effect of neighborhood.

\section{Conclusion}

HEDM is a unique probe enabling scientists and engineers to view features in materials that are invisible to the naked eye and to traditional microscopy methods. In its near-field configuration, a 3D crystallographic orientation map can be obtained. In the far-field configuration, the center of mass, crystallographic orientation, and lattice strain tensor of individual grains in a polycrystalline aggregate can be mapped. The evolution of crystallographic orientation and strain can be tracked on a grain-by-grain basis while the aggregate is subject to a known stimuli. The formation of substructures, phase transformations, or the introduction of damage from external stimuli can be examined on a grain-by-grain basis through analyzing the evolution of diffraction spots.

In this article, the FF-HEDM technique and its implementation at the APS 1-ID beamline have been described. Two FF-HEDM examples illustrate how this technique can contribute to our understanding of structure-property-processing relationship and provide data for validating complex material models.

\section{Acknowledgments}

This research used resources of the Advanced Photon Source, a U.S. Department of Energy (DOE) Office of Science User Facility operated for the DOE Office of Science by Argonne National Laboratory under Contract No. DE-AC02-06CH11357. Work by Zhang et al. [19] was supported by the U.S. Department of Energy, Office of Nuclear Energy, for the Nuclear Energy Enabling Technology (NEET) Program under Contract DE-AC02-06CH11357. Work by Wong et al. [23] was provided by the US Department of Energy, Office of Basic Energy Sciences, Materials Sciences and Engineering Division under Grant No. DE-FG02-10ER46758.

\section{References}

[1] SN Monteiro and S Paciornik, JOM 69(1) (2017) 84-92.

[2] E Maire and PJ Withers, Int Mater Rev 59(1) (2013) 1-43.

[3] RE Newnham, Properties of Materials; Anisostropy, Symmetry, Structure, Oxford University Press, New York, 2004.

[4] HF Poulsen, J Appl Crystallogr 45(6) (2012) 1084-97.

[5] J Budai et al., Thin Solid Films 516(22) (2008) 8013-21.

[6] R Suter et al., J Eng Mater Technol 130(2) (2008) 021007-021007-5.

[7] W Ludwig et al., Rev Sci Instrum 80(3) (2009) 033905.

[8] U Lienert et al., JOM 63(7) (2011) 70-77.

[9] A Beaudoin et al., Acta Mater 61(9) (2013) 3456-64.

[10] PA Shade et al., Rev Sci Instrum 86(9) (2015) 093902.

[11] M Varlioglu et al., Texture, Stress, and Microstructure 2010 (2010) article ID 910793, 1-10.

[12] J Oddershede et al., J Appl Cryst 48(3) (2015) 882-89.

[13] SD Shastri et al., J Synchrotron Rad 14 (2007) 204-11.

[14] A Said and S Shastri, J Synchrotron Rad 17 (2010) 425-27.

[15] SD Shastri et al., Proc SPIE 9207, Advances in X-Ray/EUV Optics and Components IX 920704.

[16] PF Tavares et al., J Synchrotron Rad 21 (2014) 862-77.

[17] X Zhang et al., Rev Sci Instrum 88(1) (2017) 01511.

[18] U Lienert et al., Mater Sci Eng A 524(1-2) (2009) 46-54.

[19] J-S Park et al., Synchrotron Radiation News 30(3) (2017) 9-16.

[20] SD Shastri, J Synchrotron Rad 11(2) (2004) 150-56.

[21] W Pantleon et al., Mater Sci Eng A 387389 (2004) 339-42.

[22] J Lee et al., Nuclear Instruments and Methods in Physics Research A 582(1) 182-84.

[23] J-S Park et al., J Mater Res 30(9) (2015) 1380-91.

[24] HO Sørensen et al., Zeitschrift für Kristallographie Crystalline Materials 227(1) (2012) 63-78.

[25] JV Bernier et al., J Strain Anal Eng 46(7) (2011) 527-47.

[26] Argonne National Laboratory, Advanced Photon Source, "MIDAS, Microstructural Imaging using Diffraction Analysis Software” https://www1.aps.anl.gov/Science/ Scientific-Software/MIDAS (accessed July 24, 2017).

[27] JM Wozniak et al., "Big data staging with MPI-IO for interactive $\mathrm{x}$-ray science," IEEE/ACM International Symposium on Big Data Computing, London, UK, Dec. 2014.

[28] X Zhang et al., J Nucl Mater 471 (2016) 280-88.

[29] J Busby, “Advanced materials for nuclear reactor systems: Alloys by design to overcome past limitations," International Conference on Fast Reactors and Related Fuel Cycles: Challenges And Opportunities (Fr09), Dec. 2009, Kyoto, Japan.

[30] PJ Maziasz, JOM 41(7) (1989) 14-20.

[31] R Swindeman and P Maziasz, "The mechanical and microstructural stability of austenitic stainless steels strengthened by MC-forming elements," International Conference on Creep of Materials, Orlando, FL, May 1992.

[32] DC Pagan and MP Miller, Acta Mater 116 (2016) 200-11.

[33] SL Wong et al., Comp Mater Sci 77 (2013) 456-66. 